\title{
Effects of TangWang prescription intervention in type 2 non- proliferative diabetic retinopathy邓a study protocol for a randomized controlled trial
}

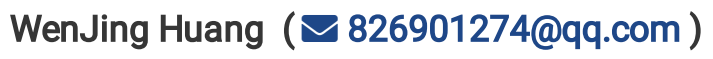

Guang`anmen Hospital https://orcid.org/0000-0001-7713-6406

De Jin

China Academy of Chinese Medical Sciences Guanganmen Hospital

\section{Xiang Meng}

China Academy of Chinese Medical Sciences Guanganmen Hospital

\section{Fan Yang}

China Academy of Chinese Medical Sciences Guanganmen Hospital

\section{Yu-jiao Zheng}

China Academy of Chinese Medical Sciences Guanganmen Hospital

\section{Qi Bao}

China Academy of Chinese Medical Sciences Guanganmen Hospital

\section{Mei-zhen Zhang}

China Academy of Chinese Medical Sciences Guanganmen Hospital

\section{Ya-nan Yang}

China Academy of Chinese Medical Sciences Guanganmen Hospital

\section{Feng-Mei Lian}

China Academy of Chinese Medical Sciences Guanganmen Hospital

\section{Xiao-Lin Tong}

China Academy of Chinese Medical Sciences Guanganmen Hospital

\section{Qing Ni}

China Academy of Chinese Medical Sciences Guanganmen Hospital

\section{Study protocol}

Keywords: Diabetic retinopathy, TangWang prescription, randomized controlled trial, traditional Chinese medicine

Posted Date: July 22nd, 2019

DOI: https://doi.org/10.21203/rs.2.11790/v1

License: (c) (1) This work is licensed under a Creative Commons Attribution 4.0 International License. Read Full License 


\section{Abstract}

Background: Presently, the efficacy of drug treatments for diabetic retinopathy (DR) is not satisfactory. This study is designed to provide evidence that a traditional Chinese medicine (TCM) formulation delays the development of DR. Methods: This study is a randomized, double-blind, placebo-controlled parallel-group, multicenter clinical trial, consisting of 384 participants to be randomized in a 1:1 ratio in the treatment and control groups, and receive a basic treatment. Furthermore, the treatment and control groups will be administered the TangWang prescription and the placebo, respectively, each at a dose of one bag twice a day. The study period will last for 48 weeks. The primary outcome measure will be the changes in the degree of retinal microvascular lesions before and after treatment. The secondary outcome will be changes in the degree of hemangioma, microvascular bleeding, microvascular leakage, macular edema, and vision. All statistical tests will be two-sided, and a $\mathrm{P}<0.05$ will be considered statistically significant. Discussion: We hypothesize that the patients with DR will benefit from TangWang prescription, and in addition to the central random system and platform of dynamic information collection, the patients' conditions will be monitored and the data collected for analysis. If successful, this study will provide evidence that the TCM formulation delays the development of DR. Trial registration: ClinicalTrials.gov, NCT03025399. Registered on January 19 2017. Keywords: Diabetic retinopathy, TangWang prescription, randomized controlled trial, traditional Chinese medicine

\section{Background}

Diabetic retinopathy (DR) is one of the most common and serious microvascular complications of diabetes mellitus (DM). In 2013, according to global estimations, the prevalence of DM was $8.3 \%[1]$ while that in China was $11.6 \%$ in adults and the number of patients was over 113 million, with the highest ranking worldwide.[2] Furthermore, DM shows an increasing trend yearly.[3-4] Epidemiological studies have found that with a DM duration of 3, 5, 10, and 15 years, the incidence of DR was $8,25,60$, and $80 \%$, respectively. In the early stage of DR, vision is not affected, but once the proliferative phase commences, irreversible visual impairment occurs. [5-7]

DR has become the leading cause of vision loss and blindness in adults. [8] The International Diabetes Control and Complications Study and UK Prospective Diabetes Study show that glycemic control effectively delays the occurrence and development of microvascular complications in type 2 DM (T2DM). [9-10] Blood pressure, blood lipid, pregnancy, inflammatory markers, and body mass index are important risk factors for the development of DR. [11-12] Presently, clinically, laser treatment is used for proliferative DR (PDR) and although it can prevent vision loss, it does not improve vision and if not properly applied may narrow the vision field.

In the non-proliferative DR (NPDR) stage, drug intervention not only effectively delays the progress of DR and improves visual function, but it also reduces the adverse effects of laser treatment. [13] Therefore, the progressive treatment of DR to reduce its incidence and prevent blindness is important in the early stages. Patients with DM having metabolic disorders should be managed to ensure blood glucose control and complications and may require drugs such as antihypertensives, lipid-lowering, and circulation improving agents.

Traditional Chinese medicine (TCM) compound preparations complement each other and can treat DR in many ways, therefore, they have development potential. Currently, in western medicine, calcium dobesilate is commonly used to treat DR, but it has general effects. TCM has unique advantages in the treatment of DR by delaying the occurrence and development of DR, as well as improving eyesight and the overall condition and quality of life of patients.

\section{AIM}

According to Professor Tong Xiaolin, TangWang prescription(TWP) is composed of five Chinese herbal medicines, and has the effect of boosting Oi, nourishing Yin, and promoting blood circulation for removing blood stasis. The main aim of 
this proposed study is to evaluate the therapeutic potential of TangWang prescription to improve the degree of retinal microaneurysm of patients with NPDR. Furthermore, the secondary aim is to evaluate the therapeutic effect of TangWang prescription in delaying the disease progression of patients with NPDR and improving the microhemangioma, microvascular bleeding, and microvascular leakage.

\section{Methods}

\section{Study design}

This study is designed as a randomized, double-blind, placebo-controlled parallel-group, multicenter clinical trial. This trial is planned to test the hypothesis that TangWang prescription can alleviate the clinical symptoms of DR and improve the state of the eyes. A total of 384 participants will be randomly divided into two groups, which will both be administered basic treatments, consisting of oral calcium dobesilate capsules (Ebewe Pharma Ges.m.b.H.Nfg KG, $0.5 \mathrm{~g}$ three times per day). Study group will be received intervention with oral calcium dobesilate capsules + TangWang prescription and control group will be treatment with oral calcium dobesilate capsules + placebo (consist of maltodextrin, starch and one-tenth the dosage of TangWang prescription as well as smell, color and texture are identical with TWP ). The study Furthermore, diabetes education and diet, as well as rational control of blood glucose, lipids, and pressure will be performed according to the American Diabetes Association guidelines. Participants will be randomly assigned to either the treatment group to receive TangWang prescription (one bag twice a day) or the control group to receive the same dosage of the placebo (Sichuan New Green Pharmaceutical Technology Development Co., Ltd.).The central randomization system uses a randomly permuted block design, which includes subject screening, randomization, emergency exposure of blindness, designated drug, drug supply, and other functions. The treatment duration is set at 48 weeks and the study medications will be labelled by pharmacists according to the randomized list prepared by the statistician involved in this study. The trial schematic flow is illustrated in Fig. 1.

In the study, the researchers, participants, staff in charge of data collection, clinical research associates, and the personnel analyzing the results will be blinded to the group assignments to avoid bias. All Chinese herbal medicines with similar efficacy to that of the study drugs will be prohibited during the study period while all co-therapies (treatment measures or medicines for other diseases) will be recorded in the combined medication table.

\section{Ethics}

The study will be conduct in accordance with the guidelines of the Declaration of Helsinki. The study protocol and conduct of the trial have been approved and will be supervised by the Ethics Committee of Guang'anmen Hospital of the China Academy of Chinese Medical Sciences (2016-092-KY-01). Central ethical approval has been confirmed from the Ethics Committee of Guang'anmen Hospital of the China Academy of Chinese Medical Sciences (No.2016-092-KY-01) and we will not begin recruiting at other centres in the trial until local ethical approval has been obtained. Study physicians will explain all the benefits of the study and known risks to each patient before enrollment in this study, and will ensure that each participant signs an informed consent form before the randomization. The following eight clinical centers in China will participate in the trial: Guang'anmen Hospital of China Academy of Chinese Medical Sciences, The First Affiliated Hospital of Anhui University of Traditional Chinese Medicine, Hubei Hospital of Traditional Chinese Medicine, Zhengzhou City Hospital of Traditional Chinese Medicine, Baoding City Hospital of Traditional Chinese Medicine, Zi bo wanjie Cancer Hospital, Shijiazhuang City Hospital of Traditional Chinese Medicine, and Zouping Country Hospital of Traditional Chinese Medicine. Data management and statistical analyses will be performed by independent data handlers and data analysts at the Institute of Basic Research in Clinical Medicine, China Academy of Chinese Medical Sciences. All the participant will be required to personally sign and date an informed consent document before randomization. Furthermore, the physician must inform patients who are of child-bearing age of the potential risk to a fetus during the study period. Furthermore, 
they would be required to take contraceptive measures to participate in this study, otherwise they would be excluded from participation.

\section{Sample size calculation}

According to preliminary research data [14], the rate of retinal microvascular disease was reduced by $3.6 \%$ in the placebo group and we predicted that the rate of retinal microvascular disease would be reduced by $8.9 \%$ in the TangWang prescription group (based on the curative effect of 10 piece on the TangWang prescription treatment group). Therefore, the sample content formula was estimated using two overall rate hypothesis tests: Assuming that $a=0.05$ and $\beta=0.10$ according to the one-sided test, to obtain the tabular value, $u a=1.64485$ and $u \beta=0.84162$, the data will be placed in the formula to obtain an $n=166.82$. Therefore, each group needs 167 participants, and considering a drop-out rate of no more than $15 \%$, each group would needs 192 participants, for a total of 384 participants.

Furthermore, the rate of retinal micro aneurysms disease increased by $3.6 \%$ in the placebo group, and according the above formula, we would need $n=108.78$ patients. Therefore, each group requires 109 participants, and if we assume a drop-out rate of no more than $20 \%$, each group needs 132 participants for a total 264 participants. Based on these two conclusions, 384 participants will be included this study.

\section{Recruitment methods}

\section{Participant screening}

Outpatient facilities for subject recruitment will be established in eight centers for the trial, where eligible patients with DR will be recruited. The Department of Endocrinology hospital medical record information will be screened to identify eligible participants with DR and telephone follow-up will be conducted. Patients with DR will be recruited through community outreach and advertisements.

\section{Staff election and training}

Doctors and camera personnel for fundus photography in each center will be interviewed and authorized by the Group Leader, and must accept to undergo unified training on standard operating procedures of relevant examinations, and collection of blood, urine, and shit save, as well as transport and filling in the specifications for medical records and use of central stochastic system.

\section{Study eligibility}

\section{Inclusion criteria}

1.Study participants must meet the diagnostic criteria of DR [18], and the required degree of disease is NPDR. [19]

(1) Mild NPDR: microaneurysms only

(2) Moderate NPDR: one or more of the following:

i. Hemorrhage $(\mathrm{H}) /$ microaneurysms moderate in 4-5 fields;

ii. or severer in one-field (or) definite intraretinal microvascular abnormality (IRMA) in 1-3 fields.

(3)Severe NPDR: one or more of the following:

i. More severe than moderate NPDR 
ii. Severe $\mathrm{H} /$ microaneurysms in $4-5$ fields

iii. IRMA $\otimes$ moderate in one-field

iv. Definite venous beading (VB) in 2-3 fields.

2.The participants must be between 30 and 70 years old.

3.The participant must sign informed consent forms.

Participants who have only one eye can meet the inclusion criteria, and the more severely damaged eye will be chosen when both eyes meet the inclusion criteria. Furthermore, the eye with a better clinical condition will be chosen when both eyes of the participant are at the same clinical stage.

\section{Exclusion criteria}

1.Patients with retinal photocoagulation, one or two eyes at the PDR phase, type I DM, other eye disease complications (such as glaucoma and cataracts, which could significantly interfere with the fundus examination, non-diabetic retinopathy, uveitis, amotio retinae, optic nerve diseases, and high myopia with fundus lesions).

2. Combined severe primary diseases such as cardiovascular, liver, kidney, or hematopoietic system; serum transaminase levels more than double the normal value; serum creatinine greater than the upper limit of normal value; and patient with psychiatric conditions.

3. Pregnancy, preparing for pregnancy, or lactating.

4. Patients participating in other clinical research studies and use of other drugs within a month of the trial.

5. Patients who have taken other drugs to treat DR except for calcium hydroxide within a week of the study.

6. Systolic blood pressure $>160 \mathrm{mmHg}$ or diastolic blood pressure $>100 \mathrm{mmHg}$.

7. Patients with diabetic ketosis, ketoacidosis, and severe infections within a month of the study.

8. Patients who have abused alcohol, used psychoactive substances, abused drugs, or experienced drug dependence within 5 years of the study.

9. According to the researcher's judgment, patients with other diseases or conditions that might reduce study compliance or complicate their participation, such as frequent changes in work environment, unstable living environment, and similar factors that could cause loss of contact.

\section{Drop-out criteria}

1. Participants whose eyes progress to proliferative lesions or undergo laser photocoagulation.

2. Participants whose vision decreases by three or more lines.

3. Participant with certain complications or special physiological changes, which are not suitable for further study. Patients who experience some complications or physiological changes rendering them unsuitable for further participation.

4. Participant with poor study drug compliance of $<80 \%$ of the requisite amount or $>120 \%$ of the prescribed amount of medicine. 
5. Participants who break blinding or emergency unblinding of a participant's information.

6. Participants who use drugs prohibited by the study plan.

\section{Voluntary withdrawal of participant}

1.Participant who do not express the desire to withdraw from the study but cease to accept medication and testing are considered withdrawn.

2.Participants who cannot continue to participate in clinical research for personal reasons; perceive poor efficacy; exhibit intolerance due to adverse reactions; and for economic reasons.

*Irrespective of the reason for withdrawal, the case record will be retained. The final test results will be used in the final analysis, and the curative effect and adverse reactions will be analyzed in the full dataset.

\section{Randomization and data collection}

The central randomization system (http://www.tcmcec.net/crivrs/.) and electronic case report form (eCRF) were established by the Clinical Evaluation Center of the Chinese Academy of Traditional Chinese Medicine. All data will be recorded by the investigator in a CRF and eCRF. Participants will be randomized to the groups, and the probability of participants being assigned to each group or center is not fixed, but will be adjusted according to certain conditions. The conditions are designed to effectively ensure that the number of participants in the various centers and important prognosis factors are similar.[15,16] According to their basic information, each participant will have a unique study subject identifier, consisting of six digits (the first and last three will be the center and group number, respectively).

Furthermore, a unique random allocation is assigned using a system, involving double-blinding for participants and researchers. Every follow-up will be required to be logged into the central stochastic system by the investigator and into the central random system to obtain a drug number. The same platform will be used to collect information on the participants, random distribution, and assigned drugs to ensure random and blind methods are implemented and the test results are objective, to improve the quality of the clinical trial [17].

\section{Data monitoring and quality control}

The designated clinical research coordinator (CRC) will inspect and monitor the SOPs used in the research study. For the clinical research, a CRC will be appointed to regularly conduct on-site inspection visits to the hospitals to ensure strict adherence to all conditions of the research program, and check the original data to ensure consistency with the CRF. This study will be supervised by a full-time Contract Research Organization (CRO) company. The study set up will include a data audit committee to audit the research data and research SOPs. Each audit will include at least one Project Manager and one project elite.

\section{Measurements}

\section{Primary outcome}

The primary outcome of the study is the changes in the degree of retinal microaneurysm lesions before and after treatment. This will be determined according to the following scale: none, mild non-proliferative phase, moderate nonproliferative phase, severe non-proliferative phase, and proliferative phase, which are divided into aggravated, unchanged, and reduced conditions. The aggravated state is defined as retinal microaneurysm lesions with a degree of severity > grade 1 after treatment. The unchanged state was defined as a degree of retinal microaneurysm lesions before and after treatment that is unchanged, while the reduced state is defined as a degree of retinal microaneurysm lesions that is reduced by $>1$ grade after treatment. 


\section{Secondary outcome}

1. Change in the number of microhemangioma in DR.

2. Change in the area of microaneurysm bleeding in DR.

3. Change in the area of microaneurysm leakage in DR.

4. Degree of change of macular edema in DR.

5. Change in vision before and after treatment [20].

Color fundus photography, fundus fluorescein angiography, and coherent opticaltomography will be performed every $6 \mathrm{x}$ months. Furthermore, the Early Treatment Diabetic Retinopathy Study (ETDRS) international visual acuity test will be performed every month.

\section{Safety evaluation index and monitoring indicators}

The blood pressure, fasting blood glucose, vital signs, physical examinations, vision tests, electrocardiogram, liver function (alanine aminotransferase, aspartate aminotransferase, gamma-glutamyl transferase, alkaline phosphatase, and total bilirubin) and renal function (blood urea nitrogen and urine creatinine) will be monitored monthly. Glycated hemoglobin and blood lipids will be determined every 3 months) while routine eye and fundus examinations, including intraocular pressure, anterior segment, lens, and vitreous body, optical coherence tomography every 6 months, vitreous body need to be monitored to describe the conditions of opacity. The schedule of enrollment, interventions, and assessments is shown in Table.2

\section{Statistical analysis}

All statistical tests will be two-sided tests, and a $\mathrm{P}<0.05$ will be considered statistically significant. Quantitative data to calculate the number of cases will be expressed as the mean, median, standard deviation, minimum, and maximum. Qualitative data will list the frequency and percentages. The demography, other baseline characteristics, and efficacy analyses will be analyzed using the full analysis set (FAS), while the per protocol set (PPS) and the safety analysis will be performed using the safety analysis set (SS).

Quantitative data will be analyzed using the t-test/Wilcoxon rank-sum test, whereas the qualitative data will be analyzed using the X2 test/exact test. The main outcome measures will be analyzed using the X2 test for comparisons between groups, whereas secondary outcome and monitoring measures will be analyzed using the t-test/signed rank test to compare the groups. The t-test/Wilcoxon rank-sum test will be used to compare the groups. All analyses will be performed using the SAS version 9.3.

Three analysis sets will be used for the data analysis. The first is the FAS, which refers to an ideal set of subjects that are as close as possible to the principle of intentionality analysis (including all subjects randomized into the group and treated at least once). If the main variable is missing in any case, the results of the closest observation will be carried forward to substitute the absent test data. We will ensure that the number of participants in each group to be evaluated in the outcome at the end of the study is as consistent as possible before the start of the study. The second dataset is the PPS, consisting of all cases that are in accordance with the research plan. This set will consist of the data of participants with good compliance of $80-120 \%$ of the prescribed dose. The CRF contains the details of the case, and the main variables can be measured if the baseline variables are not missing and there is no major violation of the test scheme. The third dataset is the SS, which will consist of the means of all participants who receive at least one treatment after 
randomization. The demographic, other baseline characteristics, and curative effect analyses, will be performed on the FAS and PPS, while safety will be analyzed using the SS.

\section{Adverse events (AEs)}

Any adverse event (AE) will be dealt with carefully, analyzed, and measures will be taken to protect all participants. AEs will be recorded in the CRF according the situation, and the record include observations such as its duration, recurrence, and disappearance. If serious AEs (SAEs) occur, the investigator must report them to higher authorities immediately, and fill out the "Severe Adverse Event (SAE)" form. This study defines pregnancy as an SAE indicating that:

1. During the study, any pregnancy event will be recorded in the corresponding data form by the researcher according to the SAE reporting procedure within the shortest time when the pregnancy event is discovered, and follow-up the outcome.

2. During the study, any abortion, whether accidental, natural, or drug-induced will be reported as an SAE.

3. Any congenital abnormalities or birth defects of a neonatus of a study participant will also be reported as an SAE. When participants experience emergencies, the main investigator can break the blind condition according to the drug and symptoms, institute relevant measures, and record the information in the CRF carefully.

\section{Clinical research process}

At the beginning of the study, we will collect the basic medical history, diagnosis, and examination indexes of the participants. The effectiveness and physicochemical examination indexes, and other parameters of the participants will also be monitored. Furthermore, we will then collect the participants' clinical data monthly and every 3 and 6 months, according to the study design. Table 3 shows the flow of the clinical data collection in detail (" $\times$ " means that the item will be implemented).

\section{Discussion}

The pathogenesis of DR is complex, and some aspects are still unclear. Studies have indicated the involvement of free radical damage, apoptosis of pericytes and retinal vascular endothelial cells, aggregation and adhesion of inflammatory factors, abnormal hemorheology, and lipid metabolism disorders, which occlude capillaries. Furthermore, the occluded capillaries are not perfused, which exacerbates local retinal ischemia and hypoxia, leading to retinal vascular injury that promotes the occurrence and development of DR.

TangWang prescription is an experiential prescription developed by Professor Tong Xiao Lin of Guang'anmen Hospital, China Academy of Chinese Medical Sciences, which consists of Astragalus mongholicus, Guizhi, medlar, Valley grass, and raw typha pollen.

TangWang prescription is an experiential prescription, that has exhibited a certain efficacy clinically. We propose that the study participants will benefit from the prescription, and we will use an active medical service model to follow-up and track patients by dynamically collecting their clinical information. If successful, this study will provide evidence of the effectiveness of a TCM preparation in delaying the development of DR.

\section{Trial status}

Approval to proceed to the full trial was received in May 2015 and the protocol version number is 201507001-11.

Furthermore, recruitment for the main trial commenced in January 2017 and is currently ongoing. The proposed end date of the study is May 312020. 


\section{Abbreviations}

DR: Diabetic retinopathy; DM: Diabetes mellitus; T2DM: Type 2 diabetes mellitus; PDN: Proliferative diabetic retinopathy; NPDR: Non-proliferative diabetic retinopathy; TCM: Traditional Chinese medicine; AEs: Adverse events; SAE: Severe Adverse Event.

\section{Declarations}

\section{Ethical Approval and Consent to participate}

Our study has been approved by the Ethics Committee of Guang'anmen Hospital of the China Academy of Chinese Medical Sciences (2016-092-KY-01), and participants provided written consent before randomization.

\section{Consent for publication}

Not applicable.

\section{Availability of supporting data}

Not applicable

\section{Competing interests}

The authors declare that they have no competing interests.

\section{Funding}

This study is supported by the 2015 Traditional Chinese Medicine Scientific Research- "the multi-center clinical research of traditional Chinese medicine intervened in early diabetic microaneurysms complications" (NO. 201507001-11). The project did not have any role in study design, data collection, analysis and interpretation the final reports of the study.

\section{Authors' contributions}

WJH and DJ drafted the trial protocol and contributed to this work equally $\mathrm{FML}, \mathrm{QN}$, and XLT conceived and designed the study. XM and QB developed the trial design and critically reviewed the manuscript. $Y Z$ and $F Y$ critically revised the manuscript. MZZ and YNY referred and recruited the patients. All authors have read and approved the final manuscript.

\section{Acknowledgments}

The authors would like to thank all research centers participating in this trial: Guang'anmen Hospital of China Academy of Chinese Medical Sciences, The First Affiliated Hospital of Anhui University of Traditional Chinese Medicine, Hubei Hospital of Traditional Chinese Medicine, Zhengzhou City Hospital of Traditional Chinese Medicine, Baodin City Hospital of Traditional Chinese Medicine, Zi bo wanjie Cancer Hospital, Shijiazhuang City Hospital of Traditional Chinese Medicine, and Zouping Country Hospital of Traditional Chinese Medicine. We also acknowledge the contributions of the Clinical Evaluation Center of Chinese Academy of Traditional Chinese Medicine, who established the central randomization system and electronic case collection system.

\section{Author information}

1. Department of Endocrinology, Guang'anmen Hospital, China Academy of Chinese Medical Sciences, Beixiange5, Xicheng District, Beijing100053, China; 2.Beijing University of Chinese Medicine, North Ring Road11, Chaoyang District, Beijing100029 China 


\section{Reference}

[1] Guariguata L, Whiting DR, Hambleton I, et al. Global estimates of diabetes prevalence for 2013 and projections for 2035 for the IDF Diabetes Atlas. Diabetes Res Clin Pract. 2014; 103: 137-149.

[2]Xu Y, Wang L, He J, et al. Prevalence and control of diabetes in Chinese adults. JAMA. 2013; 310(9): 948-959.

[3]Wang FH,Liang YB, Zhang F, et al. Prevalence of diabetic retinopathy in rural China:the Handan Eye Study. Ophthalmology. 2009; 116(3): 461-467.

[4]Varma R, Wen G, Jiang X, et al. Prevalence of diabetic retinopathy in adult chinese American individuals:the Chinese American eye study. JAMA Ophthalmol. 2016; 134(7): 785-93.

[5]Lecaire T J, Palta M, Klein R, et al. Assessing progress in retinopathy outcomes in type 1 diabetes: comparing findings from the Wisconsin Diabetes Registry Study and the Wisconsin Epidemiologic Study of Diabetic Retinopathy[J]. Diabetes Care. 2013; 36(3): 631.

[6]Chew E Y, Davis M D, Danis R P, et al. The effects of medical management on the progression of diabetic retinopathy in persons with type 2 diabetes: the Action to Control Cardiovascular Risk in Diabetes (ACCORD) Eye Study. Ophthalmology. 2014; 121(12): 2443-51.

[7]Raman R, Gella L, Srinivasan S, et al. Diabetic retinopathy: An epidemic athome and around the world. Indian Journal of Ophthalmology. 2016; 64(1): 69-75.

[8]Azad N, Agrawal L, Emanuele N V, et al. Association of blood glucose control and pancreatic reserve with diabetic retinopathy in the Veterans Affairs Diabetes Trial (VADT). Diabetologia. 2014; 57(6): 1124-1131.

[9]Intensive blood-glucose control with sulphonylureas or insulin compared withconventional treatment and risk of complications in patients with type 2diabetes(UKPDS 33).Lancet. 1998; 352(9131): 837-853.

[10]Diabetes Control and Complications Trial Research Group,Nathan DM,GenuthS,et al. The effect of intensive treatment of diabetes on the development and progression of long-term complications in insulin-dependent diabetes mellitus. NEngl J Med. 1993; 329(14): 977-986.

[11] Frank RN. Diabetic retinopathy. N Engl JMed, 2004; 350(1): 48-58.

[12]Chen Y, Zhu XH. Advances in the pathogenesis of diabetic retinopathy[J]. International Journal of Ophthalmology. 2006; 6(2): 433-435.

[13]Zhao T, Xu Q. Clinical study of fenofibrate in the treatment of diabetic retinopathy. Clinical Medicine. 2011; 31(2): 5152.

[14]Fengmei L, Lie W, Jiaxing T, et al. The effectiveness and safety of a danshen-containing Chinese herbal medicine for diabetic retinopathy: a randomized, double-blind, placebo-controlled multicenter clinical trial[J]. Journal of Ethnopharmacology. 2015; 164:71-77.

[15]FRIEDMAN LM, FURBERG CD, DEMETS DL. Fundamentals of clinical trials[M]. 3rd ed. New York: Springer. 1998: 6975区

[16]SCOTT NW, MCPHERSON GC, RAMSAY CR, et al. The method of minimization for allocation to clinical trials[J]. ControlClin Trials. 2002; 23(6): 662-674. 
[17]Shun J, Chen JC, Liu HX, et al. Characteristics and application of central random system based on electronic clinical data acquisition platform. ChineseJournal of New Drugs and Clinical Remedies. 2010; 29(9):711-715.

[18]The American Academy of Ophthalmology series, Compilation of Ophthalmology branch of Chinese Medical Association. <Preferred Practice Pattern>. Beijing: People's Medical Publishing House. 2006: 184.

[19]The American Academy of Ophthalmology series, Compilation of Ophthalmology branch of Chinese Medical Association. <Preferred Practice Pattern>[M]. Beijing: People's Medical Publishing House, 2006: 187.

[20]Zheng XY. Guiding principle of clinical research on new drugs of traditional Chinese Medicine. Chinese medicine science and Technology Press. 2002: 312-315.

\section{Tables}

Table 1.The schedule of enrollment, interventions, and assessments 


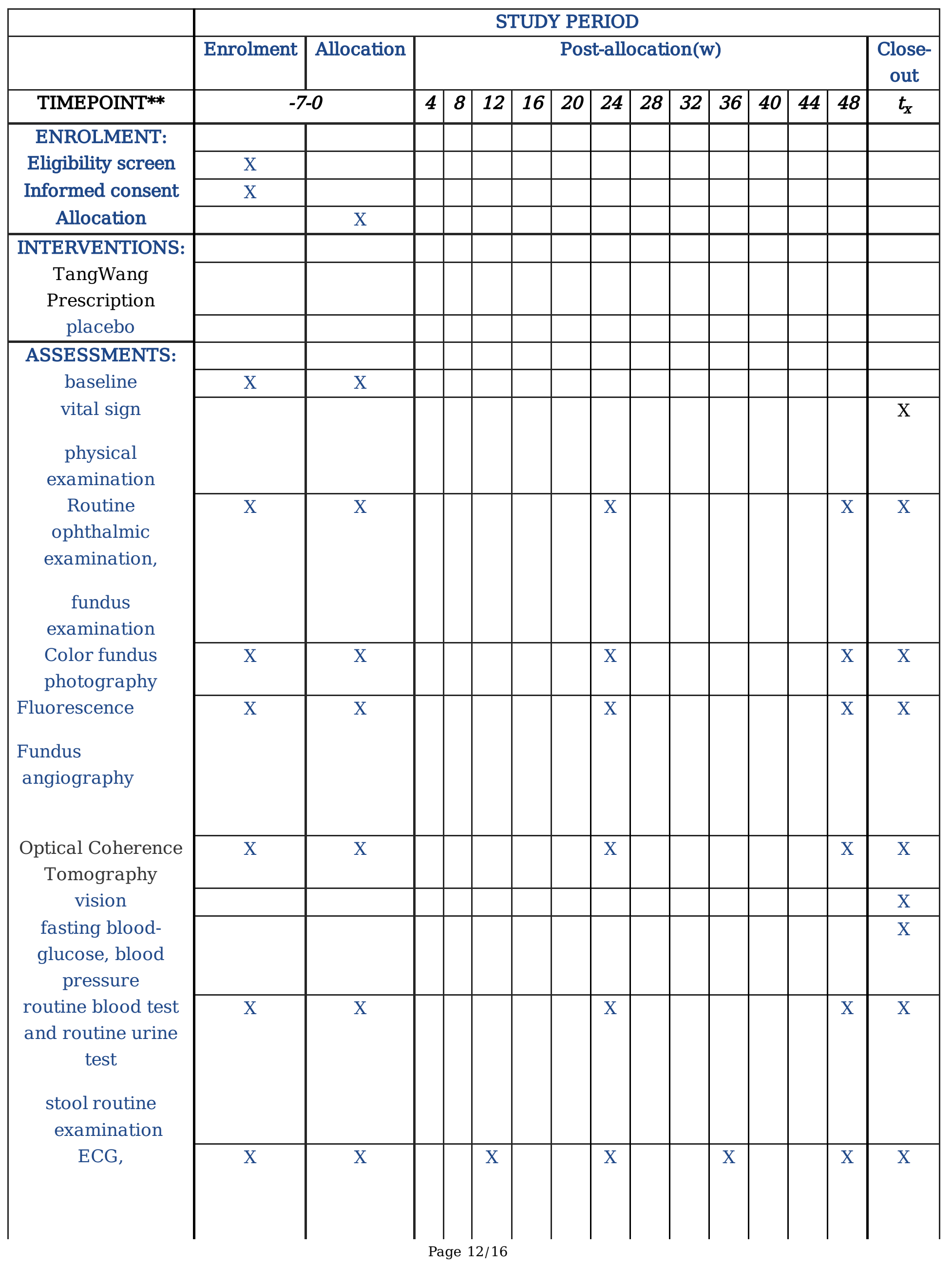




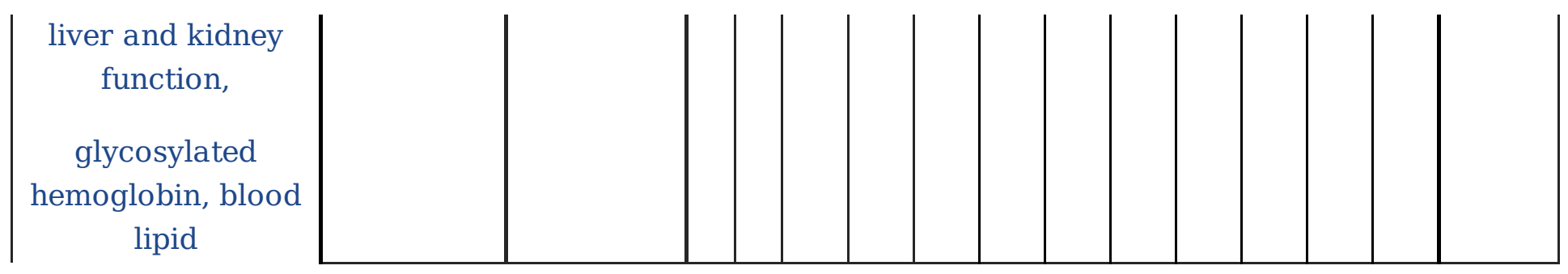

Table 2. The flow of the clinical data collection 


\begin{tabular}{|c|c|c|c|c|c|}
\hline $\begin{array}{r}\text { Project } \\
\text { Visit }\end{array}$ & $\begin{array}{l}\text { Screening } \\
\text { period } \\
\text { /baseline }\end{array}$ & $\begin{array}{l}\text { Visits } 1-2,4-5,7-8,10- \\
11\end{array}$ & Visits 3,9 & Visit 6 & Visit 12 \\
\hline Visit time & $-7-0$ days & $\begin{array}{c}\text { Medication 4, 8, 16, } \\
\text { 20, 28, } 32,40,44 \\
\text { weeks } \pm 7 \text { days }\end{array}$ & $\begin{array}{c}\text { Medication } 12, \\
36 \\
\text { weeks } \pm 7 \text { days }\end{array}$ & $\begin{array}{c}\text { Medication } \\
24 \\
\text { weeks } \pm 7 \text { days }\end{array}$ & \begin{tabular}{|c} 
Medication \\
48 \\
weeks \pm 7 days
\end{tabular} \\
\hline \multicolumn{6}{|l|}{$\begin{array}{c}\text { Collect basic medical } \\
\text { history }\end{array}$} \\
\hline $\begin{array}{l}\text { Sign informed } \\
\text { consent }\end{array}$ & $x$ & & & & \\
\hline $\begin{array}{c}\text { Fill in general } \\
\text { information }\end{array}$ & $x$ & & & & \\
\hline $\begin{array}{l}\text { History of disease } \\
\text { treatment }\end{array}$ & $x$ & & & & \\
\hline $\begin{array}{c}\text { Included inclusion } \\
\text { and exclusion } \\
\text { criteria } \\
\end{array}$ & $x$ & & & & \\
\hline vital sign & $x$ & $x$ & $x$ & $x$ & $x$ \\
\hline physical examination & $x$ & $x$ & $x$ & $x$ & $x$ \\
\hline $\begin{array}{l}\text { merge disease and } \\
\text { medication }\end{array}$ & $x$ & $x$ & $x$ & $x$ & $x$ \\
\hline \multicolumn{6}{|l|}{$\begin{array}{c}\text { Diagnosis and } \\
\text { monitoring }\end{array}$} \\
\hline urine pregnancy test & $x$ & & & & \\
\hline $\begin{array}{l}\text { fasting blood- } \\
\text { glucose, } \\
\text { blood pressure }\end{array}$ & $x$ & $x$ & $x$ & $x$ & $x$ \\
\hline $\begin{array}{c}\text { glycosylated } \\
\text { hemoglobin } \square \text { blood } \\
\text { lipids }\end{array}$ & $x$ & & $x$ & $x$ & $x$ \\
\hline $\begin{array}{l}\text { Routine ophthalmic } \\
\text { examination, } \\
\text { fundus examination }\end{array}$ & $x$ & & & $x$ & $x$ \\
\hline \multicolumn{6}{|l|}{$\begin{array}{c}\text { Observation of } \\
\text { effectiveness }\end{array}$} \\
\hline $\begin{array}{l}\text { Color fundus } \\
\text { photography }\end{array}$ & $x$ & & & $x$ & $x$ \\
\hline fluorescence fundus & $x$ & & & $x$ & $x$ \\
\hline
\end{tabular}




\begin{tabular}{|c|c|c|c|c|c|}
\hline $\begin{array}{c}\text { angiography } \\
\text { y } \\
\end{array}$ & & & & & \\
\hline $\begin{array}{c}\text { Optical Coherence } \\
\text { Tomography }\end{array}$ & $x$ & & & $x$ & $x$ \\
\hline vision & $x$ & $x$ & $x$ & $x$ & $x$ \\
\hline \multicolumn{6}{|l|}{$\begin{array}{c}\text { Physico-chemical } \\
\text { examination }\end{array}$} \\
\hline $\begin{array}{l}\text { routine blood test } \\
\text { and } \\
\text { routine urine test }\end{array}$ & $x$ & & & $x$ & $x$ \\
\hline $\begin{array}{l}\text { stool routine } \\
\text { examination }\end{array}$ & $x$ & & & $x$ & $x$ \\
\hline vital sign & $x$ & $x$ & $x$ & $x$ & $x$ \\
\hline $\begin{array}{c}\text { Electrocardiogram, } \\
\text { liver and kidney } \\
\text { function } \\
\end{array}$ & $x$ & & $x$ & $x$ & $x$ \\
\hline Adverse event & & $x$ & $x$ & $x$ & $x$ \\
\hline \multicolumn{6}{|l|}{ Other work } \\
\hline Random grouping & $x$ & & & & \\
\hline $\begin{array}{l}\text { Distribute drug and } \\
\text { Patient' diary card }\end{array}$ & $x$ & $x$ & $x$ & $x$ & \\
\hline $\begin{array}{c}\text { Recover drug, } \\
\text { quantity statistics }\end{array}$ & & $x$ & $x$ & $x$ & $x$ \\
\hline $\begin{array}{l}\text { retrieve Patient } \\
\text { 'diary card }\end{array}$ & & $x$ & $x$ & $x$ & $x$ \\
\hline Conclusion of research & & & & & $x$ \\
\hline
\end{tabular}

\section{Figures}




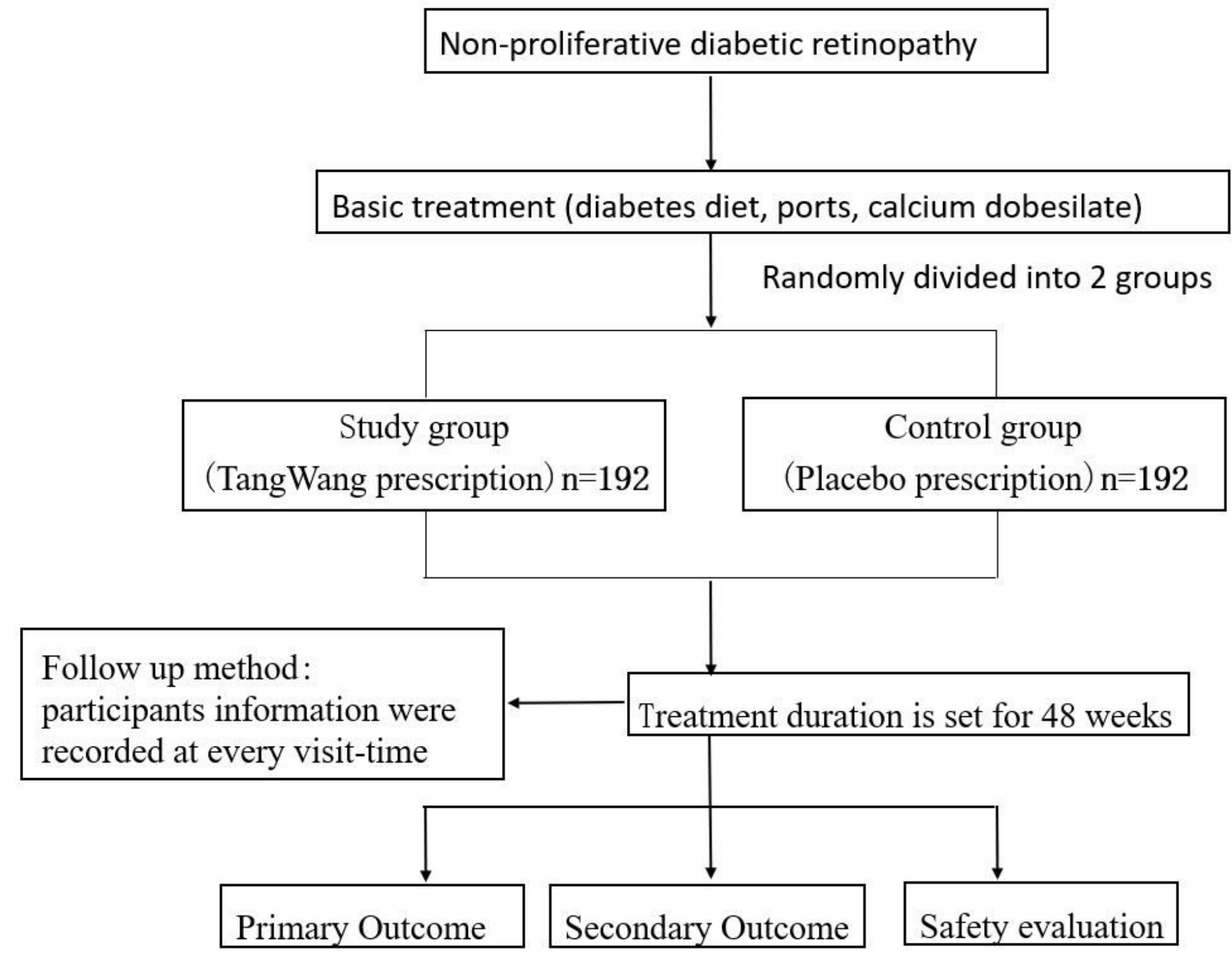

Figure 1

The trial schematic flow

\section{Supplementary Files}

This is a list of supplementary files associated with this preprint. Click to download.

- SPIRITFillablechecklist.doc 